\title{
Strates
}

STRATES Matériaux pour la recherche en sciences sociales

$11 \mid 2004$

Jeune recherche, la vitalité d'un laboratoire

\section{Le débit de boissons, cet inconnu...}

\section{Philippe GAJEWSKI}

\section{(2) OpenEdition}

\section{Journals}

Édition électronique

URL : http://journals.openedition.org/strates/407

DOI : $10.4000 /$ strates. 407

ISSN : $1777-5442$

Éditeur

Laboratoire Ladyss

Édition imprimée

Date de publication : 1 janvier 2004

ISSN : 0768-8067

\section{Référence électronique}

Philippe GAJEWSKI, «Le débit de boissons, cet inconnu... », Strates [En ligne], 11 | 2004, mis en ligne le 14 janvier 2005, consulté le 07 septembre 2020. URL : http://journals.openedition.org/strates/407 ; DOI : https://doi.org/10.4000/strates.407

Ce document a été généré automatiquement le 7 septembre 2020

Tous droits réservés 


\title{
Le débit de boissons, cet inconnu...
}

\author{
Philippe GAJEWSKI
}

1 Les débits de boissons sont des lieux importants dans l'histoire des sociétés. En Europe en particulier, là où il y a groupement humain, il $\mathrm{y}$ a ou il $\mathrm{y}$ a eu un débit de boissons. Pourtant, il reste un lieu social peu connu et mal exploité par les chercheurs, en dépit des nombreuses expériences de bistrot qu'ils relatent volontiers, hors contexte scientifique, en situation conviviale.

Bistrot, boui-boui, bastringue, rade, troquet, café, estaminet, taverne, bar à vins, pub, cyber-café, café philo et café géographique sont légalement définis comme des débits de boissons à consommer sur place. Dans nos sociétés modernes, le débit de boissons est un des lieux les plus fréquentés. Il est commun, évident, à la ville comme à la campagne. Pour certains, la connaissance du café est innée. Chacun s'est approprié un café forcément sympathique et réconfortant, d'autant qu'une culture médiatique entretient à son sujet un concept de mode de vie peut-être faussement établi. S'y rendre où y donner rendez-vous s'assimile à un réflexe. Ou bien la fermeture provisoire ou définitive d'un tel lieu public revient à une véritable perte de repères.

Beaucoup ont une tendresse avouée pour un café rural, tendresse souvent fondée sur la représentation d'une scène vécue. Ces représentations valorisent l'image populaire du café rural, affective, émotionnelle. Mais cette image ne correspond pas toujours aux références modernes en matière de mode de vie ou de morale. Elle n'est pas toujours flatteuse, elle peut être négative. Si le débit de boissons reste dans les représentations l'archétype du lieu de rassemblement, de brassage des populations et des classes sociales, si l'on clame sur tous les zincs que le café est connu de tous, il est légitime de s'interroger, d'une part, sur l'état réel de cette connaissance, et, d'autre part, sur l'intérêt de cette activité commerciale dans la vie sociale et pour la connaissance des structures et dynamiques spatiales.

4 C'est pourquoi j'ai souhaité reprendre la question sous l'angle du rôle du débit de boissons en milieu rural, en observant des milieux ruraux en France et en Écosse. Mes objectifs sont à la fois de mettre en évidence les différentes fonctions du débit de boissons et leurs adaptations aux territoires et, par le truchement de cet équipement 
commercial, de repérer des pratiques spatiales et sociales et de risquer un diagnostic de l'état des sociétés locales.

5 Je rappelerai d'abord comment les cafés ont été perçus par les sciences sociales, puis j'en indiquerai les fonctions parfois méconnues et tenterai enfin de montrer quel outil de recherche il peut être pour la connaissance des sociétés et espaces ruraux.

6 L'omniprésence, ou la quasi omniprésence des bistrots (tandis que nombre d'entre eux ont disparu), donne l'illusion que ce type d'espace commercial est très étudié. L'employer comme objet de recherche est souvent considéré "amusant» et peu sérieux. Parce qu'il est souvent lieu de rencontres théâtrales entre chercheurs et populations locales dans des circonstances occasionnellement remarquables (personnes en état d'ivresse, chants populaires, manifestations d'humour local), l'idée de faire une recherche sur le bistrot prête à sourire. Cet amusement renforce ma conviction que l'image "pagnolesque » du "café de village " est entrée sans aucune distanciation dans un modèle collectif folklorique auquel adhèrent nombre de chercheurs. Il est vrai que certains d'entre eux se font "prendre comme des touristes " dans les pièges du bistrot dont ils ignorent tout. Ils se fient à un décor "rustique typique " faussement local plus qu'au lien entre l'établissement et l'espace local vécu. Or les parasols des terrasses font plutôt fuir les habitants, car ils symbolisent l'accueil de touristes et d'étrangers à la communauté, un décorum qui attire les urbains. Les scènes du café ne peuvent-elles être objet de science ni avoir valeur scientifique? Je postule que les fonctions territoriales et sociales de ce type de commerce légalement défini comme espace public sont d'une grande valeur, et qu'il convient de les explorer au-delà des idées reçues.

7 Les formes de sociabilité spécifiques des débits de boissons en ont fait un lieu exploré par sociologues et anthropologues. Bien des sociologues français (Portet, 1984) se sont limités aux apparences, à des observations naïves des mises en scène auxquelles se livrent les habitués d'un bistrot en présence d'une personne étrangère. Ils en tirent une illustration très subjective de la sociabilité et de la cohésion sociale vues à travers le bistrot (Membrado, 1983). Pour d'autres, ces établissements renvoient à l'alcoolisme, qui n'affecte cependant pas plus de $5 \%$ de la clientèle (Bernand, 1993 ; Fortin, 1996).

Or, ce qui s'observe dans le débit de boissons donne une idée des identités locales, des modes d'habiter des populations et de leurs évolutions. Quelques historiens ont su percevoir la faculté du café à s'adapter au monde environnant : « témoin et acteur à la fois des transformations de la société, le débit de boissons change avec elle, très naturellement » (Vulic, 1991). « Le café, symbole d'ouverture sur la société englobante, l'emporte sur la veillée, facteur de repliement du monde paysan sur lui-même " (Moulin, 1988) : il demeure moderne quel que soit l'état des sociétés. Occasionnellement il est lieu de contestation et il facilite également des formes de brassage entre différentes classes d'âge et groupes sociaux.

9 Curieusement, le bistrot n'a jamais fait l'objet de recherche dans les domaines de la géographie rurale, excepté comme élément d'évaluation d'une «désertification des espaces ruraux ", fermé, comme les écoles ou d'autres services, en deçà d'un certain volume de population. Sa fonction économique a intéressé les géographes étudiant le déclin des commerces de proximité dans la France rurale (Beteille, Montagne-Villette, 1995 et Bontron, 1989).

10 La sociologie, en terme de sociabilité, s'est intéressée à ces lieux (Basas, 1990). Elle a focalisé son regard sur les formes de sociabilité internes au débit de boissons en ne se 
souciant pas de l'externe, social et spatial. La géographie peut s'y référer dans les mêmes termes, mais en portant attention à l'espace environnant, l'espace des clients consommateurs. G. Hunt ${ }^{1}$ (1986) montre qu'il est lieu de sociabilité interne à l'établissement mais que «afin de lui donner du sens, il doit être (vu comme) une continuité de la culture du groupe social externe». Si la géographie «a pour objet la connaissance de cette œuvre humaine qu'est la production et l'organisation de l'espace » (Brunet, 1993), le débit de boissons est un outil géographique, parce que son observation permet un diagnostic de l'état de la société locale et amène le chercheur à un regard sur les identités culturelles, sociales et territoriales locales, mais également sur les rapports de ces groupes à l'extérieur, à l'étranger.

11 Fréquenté par des clientèles dont la présence est facultative, le bistrot autorise la prise de parole. C'est ainsi que cafés et pubs sont des lieux de vie dans lesquels les individus se rencontrent, se racontent. Le récit public de vie, cher aux sociologues, fait partie du quotidien de ce commerce, comme, en partie, mais sur des durées plus courtes, l'épicerie ou la boulangerie... Les habitués d'un café rural ou urbain, les réguliers, ceux qui viennent quotidiennement pour retrouver « les copains » autour du zinc, ceux à qui l'on sert une boisson sans même passer commande, ceux-là ont instantanément le sentiment d'être là, à leur place, chez eux. Leur présence participe à l'élaboration du groupe, à son identité rattachée au lieu de vie plus large. Dans les débits de boissons se croisent et se complètent parcours individuels et parcours collectifs. Le "savoir » du débit de boissons est lié à cette richesse des parcours et des stratégies, collectifs ou individuels, qui donnent un sens, une réalité territoriale à la société locale.

Le territoire relève d'une lecture et d'un signifié collectifs. « Le territoire est avant tout une construction sociale. Celle-ci reflète un type de rapport à l'espace propre à plusieurs individus, à un groupe ou à plusieurs groupes » (Di Méo, 1998). Les débits de boissons participent largement à la réalisation des espaces, qu'ils soient pays ou territoires. Ainsi, élargir l'observation aux maillages qui composent son environnement, c'est utiliser le débit de boissons comme outil d'observation des sociétés et des espaces auxquels il s'adapte naturellement. Cette adaptabilité lui fait adopter différentes fonctions, différents rôles qui sont révélateurs de l'état et de l'évolution des sociétés et des territoires.

1. Espace commerçant et commerce de l'espace

13 L'enseigne et le nom usuel donnés par les habitués à un débit de boissons sont des indices utiles pour la compréhension de ses fonctions. Ce nom indique si sa fonction principale est sociale ou commerciale. Le café de la gare ou du commerce n'a pas toujours la même fonction que «chez Robert » ou "chez la mère Planquet ». Le décor spécifique n'indique pas seulement les goûts du tenancier, mais des fonctions particulières à chaque établissement. Il signe ou détermine une clientèle qui recherche tel ou tel service : capacité du commerce à l'accueillir selon sa demande qui sera selon le cas la tranquillité d'habitués, ou l'attention au passage ${ }^{2}$.

Certains débits de boissons cumulent plusieurs activités. Ils peuvent être café-épicerie, café-station service, café-poste etc. La diversification des activités commerciales internes au débit de boissons reflète la densité et la proximité des services alentour. Mais au-delà de la pluriactivité, il faudrait envisager la pluri-compétence des cafés, fonction remarquable et encore peu remarquée. 

emplacement. Un café situé dans la rue principale d'un village n'a pas la même clientèle qu'un confrère situé en retrait. Une distance, parfois réduite à quelques mètres entre deux établissements, peut représenter des décalages culturels, sociaux, techniques, économiques profonds. Par exemple, dans les villages isolés, ce sont majoritairement les anciens qui fréquentent les cafés tenus par des retraités qui maintiennent cette activité dans l'unique but d'avoir un endroit où voir les « copains ». En effet, la capacité de déplacement des anciens est limitée comparativement à celle des générations suivantes. Le café est pour eux le moyen de se rattacher à la vie locale, du village, d'une vallée. Il est aussi un moyen de retrouver une forme de liberté en bravant les « interdits médicaux » pour les malades ou les personnes de santé fragile. En milieu rural, les clients se rendent au café avec l'intention de voir le patron et prendre des nouvelles. Les villages isolés ont en effet des réseaux d'information et de connaissances restreints. Par conséquent, les patrons appartiennent au réseau personnel et quasi intime du village.

19 Faite à partir de terrains français et écossais, mon observation des cafés me permet de proposer actuellement une typologie des débits de boissons dans les espaces ruraux. Les cafés de ville ou de bourgs attractifs vivent au rythme d'espaces plus étendus, allant $\mathrm{du}$ " pays » à la nation en passant par la région. Les cafés isolés sur route de passage sont préférés des artisans, des touristes, des chauffeurs routiers, pour des commodités de stationnement. Le «territoire relationnel » de ces cafés est souvent très étendu. Les cafés de village, même sur des routes de passage, ne permettent pas d'accueillir la clientèle des cafés dits « isolés », en raison de l'absence de places de stationnement. Ces cafés accueillent une clientèle provenant d'un espace relativement étendu, mais habituée à ces lieux. Le café de village, avec une clientèle à la fois du village et de passage, est un café viable économiquement. Il favorise une clientèle locale. Le café de village, parfois très peu actif, est souvent tenu par des personnes âgées qui maintiennent leur activité pour des raisons sociales et personnelles. 
20 Un postulat courant est que les modes de vie citadins et ruraux s'influencent mutuellement (Kayser, 1995 et Di Méo, 1998). Il existe néanmoins encore des différences entre ruraux et citadins, entre jeunes et anciens dans leurs approches du débit de boissons. Les jeunes poursuivant des études toute la semaine dans les capitales régionales en adoptent les rythmes, les loisirs, et espèrent retrouver dans leurs villages ces mêmes attractions. Dans les bourgs, les patrons des cafés changent donc leur aménagement intérieur pour une décoration plus "jeune ", plus "urbaine», genre pub, taverne, bar à thèmes. Ces jeunes, par la mobilité qui les caractérise (deux roues principalement), se retrouvent dans ces cafés «modernes" qui renforcent ainsi des "pôles d'activités » par leur fréquentation. Occasionnellement, les jours de marché, les plus âgés continuent cependant de se rendre dans ces cafés transformés, qui restent dans les petites villes les seuls disponibles même s'ils ne s'y sentent plus guère « chez eux ».

21 Le samedi soir, les jeunes parcourent des distances importantes pour rejoindre le débit de boissons élu, café disposant de jeux et d'un cadre "branché ». Ce sont le plus souvent des cafés dont l'espace est prioritairement aménagé pour le tourisme. Les cafés des centres des petites villes et des bourgs sont donc alors désertés, avant de jouer le dimanche après-midi un rôle de point de ralliement coutumier. Les cafés permettent d'analyser un aspect des questions relatives aux pratiques respectives des classes d'âge en fonction de leur mobilité.

1. Des cafés et des hommes

«Les hommes ne vivent plus aussi systématiquement que jadis la coupure entre ville et campagne. Les rapports à l'espace et les formes de territorialisation qu'ils créent, s'homogénéisent d'un lieu à l'autre, du rural à l'urbain " (Di Méo, 1998). À l'inverse de cette assertion, mon observation des débits de boissons tend à montrer qu'ils sont un lieu d'attachement à la défense de l'identité locale, quelle qu'elle soit. Ils sont médiateurs des rapports sociaux en leur sein. "Lorsqu'un individu pénètre dans un débit qui ne correspond pas à son univers, à sa propre distinction, s'engage dès lors un rapport stratégique, entre lui et les formes du débit : le patron, les clients, le décor. Un rapport fait de tentatives d'intégration, ou au contraire d'exclusion, d'appropriation ou de mise à l'écart. Des stratégies qui disent, implicitement, les structures mentales, les cadres géographiques et sociaux, les intérêts particuliers ou parfois collectifs » (Vulic, 1991). Leur capacité d'adaptation leur confère une valeur de lien social. Leur observation permet de comprendre la société du village, du quartier, de territoires plus étendus.

Dans certains cas, le débit de boissons est même un lien entre culture d'entreprise locale et territoires. Certains corps de métier, après les heures travaillées, se réunissent dans des débits de boissons choisis. Des groupes d'employés, d'artisans et de retraités se retrouvent dans le bar d'un pub ou au café et échangent sur les techniques de l'entreprise et son personnel, sur les chantiers travaillés. Le café comme lieu d'interconnaissance en situation de loisir et/ou professionnelle est une forme d'espace vécu.

Mais l'observation de la clientèle, des formes de sociabilité, des distances parcourues par les " habitués " peut permettre de comprendre un territoire et ce qu'il représente pour ses habitants et ses visiteurs "étrangers ». L'émergence de groupes distincts en un même lieu peut générer une inter-surveillance «l'information sur autrui tend aujourd'hui à remplacer l'échange direct [...]. De la sociabilité aujourd'hui, nous serions tentés de dire, qu'il ne reste plus que le contrôle social qui en faisait respecter les 
règles. La société villageoise est devenue une société de «l'inter-surveillance et de l'inter-regard, faute de pouvoir produire encore l'inter-connaissance » (De la Soudière, 1981).

Les nouveaux habitants se rallient-ils au café comme epitomé du village ? L'observation méthodique du café amène à déterminer des groupes, jeunes, femmes, "anciens", nouveaux habitants, notables, et donne à comprendre des mécanismes internes à la communauté. Même s'il a la réputation de lieu de brassage, les femmes entrent rarement au café dans les espaces ruraux français, sauf dans des conditions très précises - le marché - tandis que les nouveaux habitants privilégient les salles des fêtes pour leurs activités collectives, et que les jeunes préfèrent s'échapper de lieux de proximité.

L'observation des groupes, des individus, des distances parcourues par les clients donne un inventaire sensible des territoires du quotidien. Adapté aux sociétés locales, le café met en évidence les changements sociaux, territoriaux. Pris dans l'espace rural, il amène naturellement à explorer les différentes relations locales sensibles, voire même nationales, qui irriguent l'attachement d'une population à son territoire. Par le biais du débit de boissons, on observe le dynamisme local subsistant, résistant.

Dans les espaces ruraux ou périurbains en phase de mutation, les cafés communautaires, en affirmant une identité et un système de valeurs qui leur est propre, dénoncent les mutations, ils deviennent résistants. Les habitués de ces cafés, par leur mode de sociabilité spécifique et localisé, donnent à leur bistrot l'occasion de devenir une poche de résistance sociale où les clients, en affirmant leur spécificité identitaire, ne reconnaissent pas les nouvelles formes d'utilisation de "leur» territoire. Ces cafés ultra communautaires, parfois appelés cafés d'habitués, sont perçus de l'extérieur comme des lieux sanctuarisés, fermés aux profanes à moins d'y être présenté par l'un des ses membres. Cette forme sociale communautaire du débit de boissons est remarquable, dans le sens où l'individu, membre du groupe qui accède à ce café, produit et reproduit par l'intermédiaire de micro-rituels une identité qui est propre à ce débit de boissons. C'est la reproduction de cette identité qui est un acte de résistance. Parce qu'elle permet au groupe de clients de se démarquer, de ne pas collaborer à la " pensée unique ", mais, au contraire, de diversifier les modes de penser, d'habiter. Cependant, pour ces cafés communautaires, se démarquer, reproduire une identité, c'est prendre le risque d'être perçus comme des lieux surannés, décalés.

Il résulte de cette rapide description de l'objet débit de boissons dans l'espace qu'il permet un certain diagnostic de l'état de la société et des territoires. Les études de terrain menées jusqu'alors tendent à montrer que les espaces ruraux vus au travers du café et du pub sont de très bons indicateurs quant à l'évolution des sociétés européennes et à leur devenir.

C'est en cela que le café sert l'observation du quotidien et en même temps, il vérifie que le conformisme implique l'adhésion à tout un système. Il est un outil transversal, et ses capacités d'observation des territoires et des sociétés locales semblent pertinentes pour une compréhension de la complexité des sociétés. Il n'est pas un commerce hors sol. Il est un lieu public à part, car il autorise les commentaires, les réflexions, les discussions de tout ce qui semble acquis par la société au sens le plus institutionnel du terme. Contestataire dans un esprit de construction, de conservation de valeurs locales, il est un support d'analyse pour l'étude des identités, des nouvelles façons d'habiter des espaces européens, un lieu dans lequel se révèlent les appartenances territoriales. Et 
comprendre le fonctionnement de la société, en trouver les outils, c'est aussi tourner son regard vers cet illustre inconnu.

\section{BIBLIOGRAPHIE}

Basas A., 1990, « De la sociabilité au café » Le social dans tous ses états, Paris, l'Harmattan, p. 115-123.

Bernand C., 1993, « Alcool et intégration », Cahiers de l'IREB, no 11.

Beteille R., Montagne-Villette, 1995, Le « rural profond » français, Paris, Sedes, 166 p.

Bontron J. C., 1989, « Les établissements commerciaux en milieu rural », Grand Atlas de la France rurale, Paris, De Monza, p. 72-73.

Brunet R., 1993, Les mots de la géographie, dictionnaire critique, Paris, Reclus, 522 p.

De la Soudière M., 1980, « Mobilité et sociabilité villageoise », revue POUR, p. 34-39.

Di Méo G., 1998, Géographie sociale et territoires, Paris, Nathan, 317 p.

Fortin M. alias Barthelemy, 1996, Planète café, Paris, Albin Michel, 148 p.

Hunt G. (1986) The Pub, the Village and the People, Dept sociology polytechnic North London, N5 2AD England.

Kayser B., 1995, « Une ressource nationale à valoriser », in P. Noirot (dir.), Rural : une carte pour la France, L'Acteur Rural, p. 10-17.

Membrado M., 1983, Le bistrot et l'imaginaire utopique, Thèse de sociologie, Toulouse II (directeur R. Ledrut).

Moulin A., 1988, Les paysans dans la société française, Paris, Seuil.

Portet F., 1984, « Les bistrots, ces formidables pourvoyeurs de sociabilité urbaine », Revue Milieu, no 18 , juin-septembre, p. 13-14.

Vulic A., 1991, Le cabaret, le bistrot, lieu de sociabilité populaire dans le bassin houiller du Nord-Pas-deCalais (1750-1985), Thèse d'histoire, Lille ${ }^{4}$.

\section{NOTES}

1.Sociologue britannique.

2.Les grands groupes brassicoles l'ont bien comprise : propriétaires de plus de $80 \%$ des pubs de Grande-Bretagne, ces groupes brassicoles ont créé une dizaine de thématiques appliquée à chaque pub. Ces thèmes, liés à des enseignes spécifiques, privilégient des modes d'accueil particuliers. Ainsi, de nombreux pubs portent des noms similaires qui évoquent une priorité thématique et un mode de fonctionnement spécifique. Ces thèmes commerciaux ont pour but de cibler et fidéliser au mieux une clientèle. 
3.Un pub est traditionnellement divisé en deux salles : le bar, qui est une salle réservée aux habitués et dans lequel la décoration est sommaire et le loundge qui est une salle moquettée, ouverte à tous et dans laquelle la décoration est soignée. Dans la ville de Grantown (Écosse), le barman d'un pub m'a demandé de quitter la salle du bar pour celle du loundge, seule la population locale, à certaines heures, restant du côté du bar. Étranger à la communauté, je «n'étais pas à ma place ».

4.Observation de terrain, Nord Aveyron.

\section{RÉSUMÉS}

Cafés et pubs sont des commerces omniprésents dans nos sociétés. Haut lieu social, le débit de boissons a parfois été mal exploité par les sciences sociales focalisées par les formes de sociabilité internes à l'établissement. Élargir l'observation aux maillages qui composent son environnement, c'est utiliser le débit de boissons comme outil d'observation des sociétés et des espaces auxquels il s'adapte. Cette adaptabilité lui fait adopter différentes fonctions, différents rôles, parfois méconnus, qui sont révélateurs de l'état et de l'évolution des sociétés et des territoires. Ainsi, ces commerces permettent un diagnostic de l'état de la société locale car ils sont des lieux de convergences des identités, des parcours individuels et collectifs, et sont aussi représentatifs des valeurs locales et des pratiques spatiales/sociales.

Cafés and pubs are omnipresent in our societies. Drinking establishments are well-known socializing places but they have not always been thoroughly exploited by social sciences, which have tended to focus only on the forms of sociability that develop within the place itself. Broadening our observation to the different elements of its environment allows us to use drinking establishments as tools for the observation of the societies and spaces to which they adapt. Thanks to such adaptability, they can adopt different fonctions, different roles (sometimes unknown), that reveal the state and evolution of societies and spaces. They allow to make a diagnosis of the state of the local society, because they work as places where identities as well as individual and collective paths converge, and are also representative of local values and social/ spatial practices.

\section{INDEX}

Keywords : France

Mots-clés : Espace

\section{AUTEUR}

PHILIPPE GAJEWSKI

Géographe, Doctorant, Ladyss, université Paris 8, gadjo@wanadoo.fr 